\title{
Posiciones políticas sobre el derecho al turismo
}

\author{
Political positions on the right to tourism
}

\author{
ALEJANDRO MANTECÓN (Universidad de Alicante) y RAQUEL HUETE (Universidad de \\ Alicante)
}

Artículo recibido: 26 de febrero de 2020

Solicitud de revisión: 10 de mayo de 2020

Artículo aceptado: 15 de junio de 2020

Mantecón, Alejandro, Huete, Raquel (2021). Posiciones políticas sobre el derecho al turismo. Recerca. Revista de Pensament i Análisi, 26(1), pp. 33-58. doi:

http://dx.doi.org/10.6035/Recerca.2021.26.1.3

\section{Resumen}

El objetivo de este trabajo es estudiar las relaciones que se establecen entre turismo, ideología y poder. En concreto, se quiere profundizar en la comprensión de los argumentos que legitiman o cuestionan la consideración del turismo como un derecho social. La explicación que se propone se fundamenta en la revisión de documentos publicados por organizaciones que tienen un rol significativo en el sistema turístico. Finalmente, se caracterizan y comparan las posiciones de tres bloques ideológicos: neoliberal, del capitalismo social y altermundista, que pugnan por imponer su definición de la realidad turística y su valoración de lo que debería ser.

Palabras clave: ideología, discurso, poder, turismo, sostenibilidad.

\begin{abstract}
The objective of this work is to study the relationships between tourism, ideology and power. We want to deepen the understanding of the arguments that legitimize or question the consideration of tourism as a social right. The explanation is based on the review of documents published by organizations that have a significant role in the tourism system. Finally, three ideological blocks are featured and compared: neoliberal, welfare capitalism and altermondialist, which struggle to impose their definition of tourist reality and their assessment of what it should be.
\end{abstract}

Key Words: ideology, discourse, power, tourism, sustainability. 


\section{INTRODUCCIÓN}

El turismo se ha agregado recientemente a la discusión pública como un asunto controvertido, a pesar de que la línea de estudios críticos se remonta al siglo pasado. La influencia que la digitalización de las actividades comerciales está ejerciendo en la reconfiguración del negocio del alojamiento (Gil, 2019; Huete, 2019), junto con la incorporación de la cuestión turística a la agenda reivindicativa de diferentes movimientos sociales (Milano, 2017), reubican el turismo como un tema central, expuesto a las luchas ideológicas que distintas posiciones entablan con el fin de decidir de qué - y cómo- se tiene que hablar - y pensar- en cada momento (Huete y Mantecón, 2018).

El objetivo de este trabajo es contribuir a la elaboración de un marco interpretativo con el que orientar la investigación de los principales posicionamientos políticos sobre el derecho al turismo. El hilo conductor que cohesiona la exposición problematiza una premisa elevada hoy a la categoría de ley moral y, por ello, poco discutida: la consideración del turismo como un derecho social. Lejos de asentarse en pilares unívocos, esta es una idea polémica que admite enfoques diversos.

El debate académico sobre el derecho al turismo es escaso, lo que dificulta elaborar un estado del arte sobre el mismo. Las publicaciones más relevantes se incluyen en los siguientes apartados como soporte del argumento analítico. A excepción del artículo de Breakey y Breakey (2013), que ofrece un respaldo teórico a las posiciones más legitimadoras, el denominador común es un componente crítico vinculado con las desigualdades y las contradicciones que acompañan a los desplazamientos turísticos (Bianchi, Stephenson y Hannam, 2020; Bianchi y Stephenson, 2014; McCabe y Diekmann, 2015) y con la instrumentalización del turismo como impulsor de las dinámicas más agresivas del capitalismo (Castañeda, 2012; Gascón, 2016, 2019). En un segundo plano, la cuestión también es tratada en algunos textos que exploran las dimensiones éticas del turismo. Ejemplos son los trabajos de Fennell (2019) o de LópezGonzález (2018). En el primero se analiza el carácter neoliberal del Código Ético Mundial para el Turismo promulgado por la Organización Mundial del Turismo - en adelante, OMT-. En el segundo se ponen en cuestión los supuestos cambios derivados de la transformación de dicho código — cuya aplicación dependía de la voluntad de los agentes turísticos- en una Convención Marco sobre Ética del Turismo - con un fundamento legal y, por lo tanto, de aplicación heterónoma-. 
Nuestra aportación consiste en el reconocimiento, explicación y comparación de tres grandes bloques ideológicos en competencia. Los hemos llamado neoliberal, del capitalismo social y altermundista. Sus fronteras son difusas y porosas, consecuencia del carácter reflexivo de la vida en sociedad y, específicamente, de una fluidez axiológica habitual en las estrategias políticodiscursivas. Por ello, no es raro que en distintos momentos una misma organización llegue a emitir declaraciones susceptibles de encajar en bloques diferentes.

\section{TURISMO, POLÍTICA E IDEOLOGÍA}

Si la exploración de las caras más conflictivas del turismo tiene su origen en la década de 1970 (De Kadt, 1979; Estivill, 1979; Gaviria, 1974; Greenwood, 1977; Jurdao, 1979; Nash, 1977; Turner y Ash, 1975), la mayoría de los intentos académicos por entender su incrustación en la esfera política tardaron más en aparecer. ${ }^{1}$

Una ramificación de esta línea es la que defiende una aproximación a las relaciones entre turismo y poder que trascienda los análisis politológicos ortodoxos - más preocupados por investigar el rol del Estado y de las administraciones públicas en la gestión turística- y conciba el poder como un elemento que empapa toda la estructura social. Se alude a aquellas propuestas que asocian el estudio del turismo al análisis de los discursos y las ideologías (Tribe, 2007). Ejemplos pueden hallarse en Ateljevic y Doorne (2002), Bramwell (2006), Cohen-Hattab (2004), Huete y Mantecón (2018), Mantecón (2008, 2011), Sepúlveda-Sánchez y Airey (2017), Smith (1997), van-den-Berghe y Flores-Ochoa (2000) o Webster e Ivanov (2016). En esta corriente se sitúa el presente artículo.

Por ideología no se entiende aquí un mero sistema de creencias compartido por un conjunto de personas, sino una interpretación de la realidad, lógica en apariencia, que sirve a los intereses de un grupo implicado en la misma mediante la recomendación y justificación de determinadas acciones (Berger, 1963). No se va a distinguir, como hacía Mannheim (1929), entre tipos de mentalidad ideológica —inconsciente, de autoengaño o mentirosa-. La atención

1 Una discusión de la primera literatura se recoge en Matthews y Richter (1991). Obras que contienen perspectivas críticas son las de Bramwell y Meyer (2007), Burns y Novelli (2007) o Hall (1994). Para el caso español es destacable la revisión de Aguiló y Vich i Martorell (1996), los trabajos de Pack (2006), Velasco (2016) y el pionero libro de Cals (1974). 
se centra en la identificación cualitativa de posiciones básicas y en la comparación del modo en el que articulan discursivamente su interpretación del derecho al turismo. En los discursos, argumentos socialmente construidos productores de sentido -o de regímenes de verdad, como precisaba Foucault-, se materializa la ideología y, gracias a ellos, se expande y reproduce.

Este ensayo se fundamenta en documentos redactados por organizaciones que tienen un rol significativo en el sistema turístico (véase la figura 1). No existe un registro de informes en torno al derecho al turismo. Por eso, y dado el carácter cualitativo de la aproximación metodológica, se optó por proceder a la selección de una muestra orientada por criterios subjetivos - muestreo intencional-. Dichos criterios tienen que ver en este caso con: $a$ ) la búsqueda de documentos que recogieran valoraciones sobre el derecho al turismo, y $b$ ) que estos estuvieran elaborados por organizaciones reconocidas por la emisión de análisis o declaraciones a propósito de las dinámicas turísticas. Esta lógica es similar a la seguida en otras investigaciones parecidas (Robinson, Martins, Solnet y Baum, 2019). El tamaño de la muestra se decidió tras constatar la saturación de los discursos - los últimos documentos consultados ofrecían valoraciones redundantes-.

Figura 1

Fuentes documentales

\begin{tabular}{|c|c|}
\hline Organización & Documento \\
\hline ETC (2019) & Horizon 2022: https://etc-corporate.org/our-campaigns/horizon-2022/ \\
\hline ETC (2019) & $\begin{array}{l}\text { European Tourism: Trends \& Prospects: https://etc- } \\
\text { corporate.org/uploads/2020/02/ETC_Quarterly-Report-Q4- } \\
\text { 2019_Public.pdf }\end{array}$ \\
\hline $\begin{array}{l}\text { Exceltur } \\
(2020)\end{array}$ & $\begin{array}{l}\text { Mensaje del Presidente: https://www.exceltur.org/conozca- } \\
\text { exceltur/mensaje-del-presidente-2/ }\end{array}$ \\
\hline $\begin{array}{l}\text { Greenpeace } \\
(2018)\end{array}$ & $\begin{array}{l}\text { ¡Vacaciones por fin! Pero sin destrozar el medio ambiente: } \\
\text { https://es.greenpeace.org/es/noticias/vacaciones-por-fin-pero-sin- } \\
\text { destrozar-el-medio-ambiente/ }\end{array}$ \\
\hline OCDE (2020) & $\begin{array}{l}\text { Tourism Trends and Policies 2020: } \\
\text { https://www.oecd.org/cfe/tourism/OECD-Tourism-Trends- } \\
\text { Policies\%202020-Highlights-ENG.pdf }\end{array}$ \\
\hline OIT (2008) & $\begin{array}{l}\text { Acuerdo entre la Organización Mundial del Turismo (OMT) y la Organi- } \\
\text { zación Internacional del Trabajo (OIT): }\end{array}$ \\
\hline
\end{tabular}


https://www.ilo.org/wcmsp5/groups/public/---dgreports/--jur/documents/genericdocument/wcms_434588.pdf

OIT (2011) Guía Práctica sobre la Reducción de la Pobreza a través del Turismo: https://www.ilo.org/wcmsp5/groups/public/---ed_dialogue/--sector/documents/instructionalmaterial/wcms_171733.pdf

OIT (2017) Pautas de la OIT sobre trabajo decente y turismo socialmente responsable: https://www.ilo.org/wcmsp5/groups/public/---ed_dialogue/--sector/documents/normativeinstrument/wcms_546341.pdf

OIT (2019) El turismo sostenible: un catalizador del desarrollo socioeconómico inclusivo y la reducción de la pobreza en las zonas rurales: https://www.ilo.org/global/topics/economic-and-socialdevelopment/rural-development/WCMS_601266/lang--es/index.htm

REDS (2019) Guía para un turismo sostenible. Retos del sector turístico ante la Agenda 2030: https://reds-sdsn.es/wp-content/uploads/2019/10/Gui\%CC\%81apara-un-turismo-sostenible-REDS-RTI-web.pdf

RTI (2015) Carta Mundial del Turismo Sostenible +20: http://cartamundialdeturismosostenible2015.com/

Secretaría de Directrices generales de la Estrategia de Turismo Sostenible de España

Estado de 2030: https://turismo.gob.es/es-es/estrategia-turismo-

Turismo de sostenible/Documents/directrices-estrategia-turismo-sostenible.pdf

España (2019)

Tourism Concern (2015) The Perceived Impacts of all-inclusive package holidays on host destinations: https://www.tourismconcern.org.uk/reports/

Transforming Tourism (2017)

UNEP \& Making Tourism more Sustainable: A Guide for Policy Makers: WTO (2005) http://wedocs.unep.org/handle/20.500.11822/8741

VVAA (2016) Manifiesto Europeo del Turismo para el Crecimiento y el Empleo: https://tourismmanifesto.eu/

WTTC (2019) World Transformed Megatrends and their Implications for Travel and Tourism: https://sp.wttc.org/Research/Insights

Fuente: elaboración propia

Se trabajó con una masa de textos, aislando aquellos fragmentos que permiten entender cuál es la posición de la organización acerca del derecho al 
turismo. El material textual reunido se reclasificó elaborando notas, asociando ideas y descubriendo líneas argumentales - pero no hay intención de completar un análisis en profundidad de sus dimensiones temáticas-. Esta operación se repitió de manera sistemática hasta que fue posible reconocer ciertos «patrones de regularidad» (Conde, 2009: 106). Los extractos que aparecen en las próximas páginas no poseen representatividad estadística, sino tipológica: ilustran la orientación de cada uno de los bloques mencionados.

\subsection{La posición neoliberal}

La Organización Internacional del Trabajo (OIT) aprueba en su tercera conferencia (Ginebra, 1921) un convenio para regular el derecho al descanso dominical de los trabajadores industriales. A partir de 1945 establece una serie de acuerdos de colaboración con la recién fundada Organización de las $\mathrm{Na}$ ciones Unidas (ONU). La ONU asumirá el reconocimiento del ocio como un derecho social con documentos como la Declaración Universal de los Derechos Humanos - cuyo artículo 24 explicita que «Todas las personas tienen derecho al descanso y el ocio, incluyendo una limitación razonable de las horas de trabajo y un periodo de vacaciones pagadas»- (ONU, 1948) o el artículo $7 \mathrm{~d}$ del Pacto Internacional de Derechos Económicos, Sociales y Culturales (ONU, 1966).

Sin embargo, llama la atención la relevancia que más tarde adquiere el derecho al descanso y al ocio en el artículo 7 del Código Ético Mundial para el Turismo:

La posibilidad de acceso directo y personal al descubrimiento de las riquezas de nuestro mundo constituirá un derecho abierto por igual a todos los habitantes de nuestro planeta. La participación cada vez más difundida en el turismo nacional e internacional debe entenderse como una de las mejores expresiones posibles del continuo crecimiento del tiempo libre, y no se le opondrá obstáculo ninguno (OMT, 2001).

En estas líneas el mundo es puesto a los pies del turista. Ya no es el descanso lo que se presenta como un derecho social universal, sino el acceso de toda la humanidad - sin restricciones - a una forma muy concreta de ocio activo: el viaje internacional motivado por la búsqueda de experiencias placenteras ajenas a las obligaciones propias de la esfera laboral.

La OMT es el organismo político que mejor encarna los valores de la posición neoliberal. Su cometido esencial ha sido facilitar la expansión de la indus- 
tria turística. Es una agencia internacional creada en 1975 y vinculada a la ONU desde 1976. Salvo contadas excepciones (Castañeda, 2012; Gascón, 2016, 2019; López-González, 2018), las discusiones académicas no suelen reflejar controversias significativas a propósito de la inclusión del derecho al turismo bajo el mismo paraguas político-administrativo que ampara la defensa de los fines humanitarios perseguidos por la FAO - Organización para la Alimentación y la Agricultura-, la OMS - Organización Mundial de la Salud-, el PNUD -Programa de las Naciones Unidas para el Desarrollo-, la Unesco -Organización de las Naciones Unidas para la Educación, la Ciencia y la Cultura-, Unicef -Fondo de las Naciones Unidas para la Infancia-, el UNEP - Programa de las Naciones Unidas para el Medio Ambiente- o la citada OIT.

A partir de la clasificación de los 490 miembros afiliados en junio de 2015 a la OMT, Gascón ponía de manifiesto la naturaleza de esta organización como un instrumento al servicio de intereses empresariales — más de la mitad de sus miembros pueden catalogarse como empresas o grupos empresariales privados. En este sentido, afirma: «no es extraño, por lo tanto, que el objetivo explícito de la OMT sea impulsar el crecimiento del sector, y no regularlo o controlarlo, lo que tal vez sería más apropiado para un organismo de Naciones Unidas» (Gascón, 2016: 62). Ante esta situación, Higgins-Desbiolles (2018a) llega a sugerir su refundación en una Oficina para las Movilidades Sostenibles y un Fondo Global de Riqueza Turística destinados a, por ejemplo, establecer los límites ecológicos de la actividad turística.

Un trabajo académico representativo de la línea argumentativa según la cual el envoltorio ético-jurídico debería de respaldar la expansión del negocio turístico internacional es el de Breakey y Breakey (2013). Los autores se escudan en filósofos liberales de la talla de Locke o Stuart Mill para articular un planteamiento tan simple como inconsistente: la defensa del derecho al turismo a partir del reconocimiento previo de, por un lado, el derecho al descanso $\mathrm{y}$ al ocio $\mathrm{y}$, por otro, a la libre circulación de las personas. Este razonamiento es falaz porque en la dinámica social, como en un experimento químico, la unión de dos elementos a menudo da lugar a un tercero con propiedades peculiares e independientes de las partes que lo componen. El acceso al ocio y la facultad humana de desplazarse preceden al turismo moderno - masivo-, cuyo origen está instrumentalizado por intereses empresariales que tienen poco que ver con la protección de derechos fundamentales (Goldstone, 2001).

Cuando la posición neoliberal propone una defensa del derecho al turismo, esgrime en la práctica una defensa de la capacidad del capitalismo para producir riqueza mercantilizando cada vez más elementos en cada vez más 
lugares. El discurso de los lobbies empresariales del sector turístico, como el del español Exceltur, ejemplifica esta aproximación:

El sector turístico español lleva años trabajando en reposicionar, desestacionalizar y especializar la oferta turística, vía nuevas líneas de producto como el turismo de compras, naturaleza, gastronomía o arte, y también en diversificar sus mercados y segmentos de clientes [...] No cesaremos tampoco de reclamar a las diversas Administraciones una mayor dotación de recursos presupuestarios, y minimizar las cargas impositivas y tasas que gravan indebidamente al turismo, mermando su capacidad para competir en entornos como el actual (Exceltur, 2020).

En diciembre de 2015, una veintena de las principales organizaciones turísticas europeas firmaron el Manifiesto Europeo del Turismo para el Crecimiento y el Empleo. En el primer párrafo del documento señalan:

El turismo es un importante motor de desarrollo económico y social. El sector estimula el crecimiento económico al generar ingresos, empleo e inversión en Europa y a través de sus exportaciones a mercados de origen de todo el mundo. Ayuda a mantener nuestro patrimonio cultural y natural, proporciona ingresos para financiar instalaciones e infraestructuras de las que disfrutan los visitantes y residentes y conciencia sobre la identidad común y la ciudadanía europeas distinguidas por su diversidad (Tourism Manifesto, 2015).

Entre las 44 prioridades que dan forma al núcleo del manifiesto se apunta aquí la número 36:

Apoyar el concepto de turismo como derecho de todos. Dar la posibilidad de viajar a todos los ciudadanos europeos, incluidos los ciudadanos con bajos ingresos o con discapacidad (Tourism Manifesto, 2015).

Desde mediados del siglo XX, corporaciones transnacionales - el caso de American Express es ilustrativo- publicitan el negocio turístico como impulsor de la democracia y de la paz mundial (Goldstone, 2001). Se crea así un imaginario que convierte el turismo en un punto nodal al que se unen los conceptos de progreso, modernización o libertad. El producto interior bruto es el indicador favorito de esta posición: registra la riqueza que resulta de la actividad económica sin hacer referencia a su distribución, a sus costes socioculturales o a la huella ecológica que deja impresa. 


\subsection{La posición del capitalismo social}

Con el término capitalismo social - equivalente en este texto a la expresión capitalismo de bienestar - se alude de un modo genérico a las formas de organización de la vida colectiva en las que confluyen los principios del libre mercado con la implementación por el Estado de políticas sociales más o menos intensas (Esping-Andersen, 1990).

En estos discursos el derecho al turismo no cuestiona la conveniencia de promover un derecho al consumo masivo de experiencias turísticas, pero sí problematiza el modo en el que esa promoción debe organizarse. A grandes rasgos, la atención se fija en dos asuntos: el desigual acceso al turismo y los impactos - sobre todo ambientales-que el turismo provoca.

En el 2015 se organizó en Vitoria-Gasteiz la Cumbre Mundial de Turismo Sostenible por iniciativa del Responsible Tourism Institute (RTI). La revisión del compromiso asumido en Lanzarote veinte años antes quedó reflejada en una nueva Carta del Turismo Sostenible +20 . Es un documento ilustrativo de esta posición:

En este contexto surgen los destinos turísticos inteligentes como avanzadilla del turismo sostenible del futuro. Asistimos a la aparición de este nuevo concepto de destinos turísticos innovadores, consolidados sobre una infraestructura tecnológica avanzada y ecoeficiente, que garantizan el desarrollo sostenible del territorio turístico, accesible para todos, y que facilitan la integración del visitante con el entorno, incrementando la calidad de su experiencia [...] El turismo sostenible está llamado a contribuir de forma efectiva a la mitigación de la pobreza y a la erradicación de las desigualdades en los destinos, a través de una mejor redistribución de los ingresos y la eliminación de criterios y actividades excluyentes. Entendido el turismo como un derecho, los criterios de equidad deben extenderse también a los propios turistas, fomentando acciones como el turismo inclusivo (RTI, 2015).

Existe una interesante literatura académica que relaciona el derecho al turismo con la existencia de desigualdades sociales (Bianchi, Stephenson y Hannam, 2020; Bianchi y Stephenson, 2014; McCabe y Diekmann, 2015). Desde esta perspectiva, el consumo se habría convertido en el principal mecanismo de integración informal de las sociedades capitalistas, la vía no administrativa mediante la cual se obtiene el estatus de ciudadano y, en definitiva, un medio de creciente importancia por el que las personas construyen la imagen que tienen de sí mismas. Si el turismo es un objeto de consumo masivo, quienes quedan excluidos de él contra su voluntad se verían a su vez inmersos en un 
proceso más amplio de exclusión y de deterioro de la identidad individual. Esta realidad adquiere tintes dramáticos al considerar el papel terapéutico con el que las prácticas turísticas son presentadas ante la opinión pública desde hace décadas (Gil de Arriba, 1992). Por lo tanto, siendo coherentes con los principios inspiradores del capitalismo social, el Estado tendría que asumir un papel activo facilitando el acceso a los beneficios derivados del turismo a aquellas personas - turistas y comunidades receptoras - con insuficiente capital -económico, social o cultural - o con alguna característica que de algún modo dificulta su actuación en la economía turística (Clemente, Bote y SánchezVera, 2018; Schenkel, 2018). La legitimidad del modelo de sociedad en que vivimos dependería de ello.

Respecto a los impactos del turismo sobre el medio ambiente, la OMT y miles de instituciones se han identificado con los principios esenciales del paradigma de la sostenibilidad. Las toneladas de informes que en las últimas décadas vinculan el turismo con la sostenibilidad, hasta fundirse en la expresión recurrente "turismo sostenible», muestran la dimensión que ha adquirido este discurso. Estos documentos están plagados de análisis que buscan corregir los efectos no deseados de las lógicas de actuación predominantes, pero rara vez cuestionan la viabilidad futura del negocio turístico. Un ejemplo son las Directrices generales de la Estrategia de Turismo Sostenible de España 2030:

El Gobierno tiene la responsabilidad de reorientar el modelo turístico español hacia la sostenibilidad, no como un valor añadido, sino como requisito para que el turismo pueda continuar siendo un motor de desarrollo económico y social para España durante muchos más años, y que lo haga de manera en la que todo el país pueda contribuir y beneficiarse de una industria global e imparable [...] Es necesario gestionar el turismo como un motor de desarrollo económico y social, y velar por que la riqueza, los beneficios y también las cargas generadas por el turismo, se distribuyan con más equidad en todo el territorio y en todos los sectores de la ciudadanía [...] El objetivo de la nueva Estrategia de Turismo Sostenible de España 2030 es sentar las bases de la transformación del turismo español hacia un modelo de crecimiento sostenido y sostenible, que nos permita mantener su posición de liderazgo mundial. El nuevo modelo, estará apoyado en la mejora de la capacidad competitiva y rentabilidad de la industria, en los valores naturales y culturales diferenciales de los destinos, y en la distribución equitativa de los beneficios y las cargas del turismo (Secretaría de Estado de Turismo de España, 2019).

Otro texto significativo es el documento de la OIT titulado «El turismo sostenible: un catalizador del desarrollo socioeconómico inclusivo y la reducción de la pobreza en las zonas rurales», en el que se indica: 
Son necesarias políticas adecuadas para lograr un turismo sostenible que favorezca el desarrollo económico, la creación de empleo y la reducción de la pobreza sin consecuencias negativas para el destino. Dichas políticas deben fundamentarse en los principios del desarrollo sostenible y comprender la inclusión social, la conservación del entorno natural y la cultura y la promoción del trabajo decente (OIT, 2019).

Los trabajos sobre el turismo sostenible se ven influenciados por el Informe Brundtland, publicado en 1987 por la Comisión Mundial sobre el Medio Ambiente y el Desarrollo de la ONU, donde se define el desarrollo sostenible como aquel «que satisface las necesidades de la generación actual sin comprometer la capacidad de las generaciones futuras para satisfacer sus propias necesidades». Mientras provocaba los mayores riesgos socioambientales de origen antrópico nunca conocidos, la generación de los baby boomers se arrogaba el derecho de constituirse en protagonista de una sociedad de consumo con repercusiones a escala planetaria y, también, de determinar cuáles son las necesidades de su generación y de las venideras. De tal forma, las consideraciones relativas a la legitimidad moral de la definición apuntada, así como las concernientes a su operatividad analítica, se insertan en discusiones más amplias acerca del tipo de sociedad en la que vivimos y en la que nos gustaría vivir.

La ambigüedad inherente al significado del desarrollo sostenible y el uso interesado que han hecho de la expresión distintos grupos ha favorecido que, con los años, se aproxime más a un concepto político y mercadotécnico que a uno científico (Igoe, Neves y Brockington, 2010), proyectándose esa degradación conceptual en las discusiones sobre la sostenibilidad del turismo (Blázquez, 2014). Al respecto, resulta de interés comprobar cómo algunos estudios científicos (Farreny et al., 2011; Tejedo, Pertierra, Benayas y Boada, 2011; Pardo-Abad y Nieto-Codina, 2015) analizan los efectos de las dinámicas turísticas en la Antártida al tiempo que subrayan la conveniencia de gestionar del modo más sostenible los flujos de visitantes. Al menos un apriorismo es asumido por estos autores: no se plantean la posibilidad de excluir a la Antártida del sistema turístico. En este caso, no hay manera de justificar los impactos negativos que la actividad turística pueda causar en el medio natural con los beneficios económicos que pueda reportar a las comunidades receptoras - porque en la Antártida no existe tal cosa-. Las razones, entonces, habría que buscarlas en una suerte de pragmatismo sustentado en la premisa de que es imposible evitar que una parte de la humanidad incluya a la Antártida entre sus objetivos turísticos. Esa inevitabilidad se asienta en una idea compartida con la posición neoliberal: no se puede restringir la libertad de movimiento del ser humano. 


\subsection{La posición altermundista}

Los discursos altermundistas comparten su preocupación por cuestiones parecidas a las que interesan a la posición anterior, pero la forma de abordarlas cambia, si bien se advierten puntos de unión con los enfoques más críticos del capitalismo social. El rasgo distintivo de este bloque tiene que ver con el hecho de que la implementación de las políticas de decrecimiento que defiende sería, al menos en sus versiones más extremas, incompatible con la persistencia de la mayor parte de las realidades reconocibles en el actual sistema turístico. La viabilidad de las prácticas turísticas dominantes se pone en entredicho, extendiendo ese cuestionamiento a la capacidad del sistema capitalista para sostener cualquier modelo de sociedad basado en la justicia social y ambiental (Blázquez, 2014). La lógica es la siguiente: el capitalismo no es compatible con el decrecimiento y, sin políticas de decrecimiento, las condiciones de vida de la humanidad empeorarán (Foster, 2011). Las prácticas turísticas mayoritarias serían una expresión del modelo político-económico hegemónico y de sus contradicciones:

El modelo turístico convencional no es socialmente justo. Los grandes resorts promueven un modelo turístico desvinculado del entorno, proporcionan servicios subcontratados a través de empresas externas que ofrecen condiciones precarias a sus empleados. A veces, la masificación turística puede incluso llegar a convertir la ciudad en un espacio inhabitable para la ciudadanía local, ya que además de la propia sobrepoblación, provoca el encarecimiento de los bienes de consumo. Pero no está todo perdido, cada persona tenemos la capacidad y la responsabilidad de decidir qué modelo de turismo queremos consumir (Greenpeace, 2018).

Si se admite que el turismo es bueno, también se debe aceptar la conveniencia de que el acceso al mismo sea universal. Para el discurso altermundista no es posible asumir semejante premisa, pues las tendencias turísticas predominantes no pueden convivir con propuestas que aboguen por criterios serios de justicia social y conservación del medio ambiente. En la mayoría de los casos, los efectos de arrastre positivos que supuestamente acompañan a la actividad turística tenderían a convertirse en meros objetivos deseables, cuya consecución práctica se topa con serias dificultades que impiden su realización, tal y como puede apreciarse al analizar los efectos de la industria turística global en el (mal)desarrollo de las economías agrarias locales (Gascón y Ojeda, 2014; Gascón y Cañada, 2017), la destrucción de ecosistemas (Rutty, Gössling, Scott y Hall, 2015; UNEP, 2019), la vulgarización de las culturas recep- 
toras de turistas (Goldstone, 2001; Greenwood, 1977; Picard, 1996), la precarización de las condiciones laborales de quienes trabajan en el sector (Artini, Nilan y Threadgold, 2011; Cañada, 2018) o configurando economías ilícitas que, en los casos más patéticos, promueven la explotación sexual (Jeffreys, 1999; Panko y George, 2012). Hay espacios socioeconómicos en los que la benéfica «mano invisible» de Adam Smith actúa con tal sigilo que, desde esta posición, se empieza a dudar de su existencia:

El paradigma del crecimiento se basa en la suposición de que la población local se beneficia del auge del turismo a través de efectos de «derrama» y «goteo hacia abajo». Sin embargo, no incluye cómo los grupos marginados pueden hacer uso eficaz de sus derechos a la participación. Hoy en día, es ampliamente reconocido incluso por instituciones como el Fondo Monetario Internacional que la teoría del goteo no se materializa en el «mundo real» y que la continua fe en el «goteo» ha dado lugar a efectos adversos del progreso, especialmente de los grupos pobres y marginados, porque está contribuyendo a aumentar las desigualdades (Transforming Tourism. Tourism in the 2030 Agenda, 2017).

El discurso altermundista no niega que la industria turística genere beneficios, lo que subraya es que estos suelen concentrarse en grupos minoritarios especializados en la externalización de los impactos negativos del turismo sobre otros sectores sociales y en la exclusión de la sociedad civil de los procesos de toma de decisiones:

La participación de las comunidades locales es esencial para proteger sus intereses y aumentar la transparencia y la rendición de cuentas en el desarrollo turístico. Pero en la práctica sigue siendo un concepto abstracto. La gobernanza central y los enfoques descendentes prevalecen en la formulación de políticas, la planificación y la toma de decisiones. Por lo general, las empresas dominan a través de sus mecanismos de cabildeo (Transforming Tourism. Tourism in the 2030 Agenda, 2017).

Pero el principal problema tiene que ver con la relación entre el turismo y el medio ambiente. Sería incongruente que, en la fase de desarrollo tecnológico y de comercialización de vehículos consumidores de energías contaminantes y de recursos naturales no renovables en la que hoy se halla inmersa la industria de los transportes, se defendiese la sostenibilidad de una economía turística que - hasta el colapso provocado en el 2020 por la crisis de la COVID19- no había dejado de crecer, registrando 1500 millones de desplazamientos internacionales en el 2019. El derecho al turismo se convertiría entonces en un eslogan al servicio del marketing turístico. 
Los objetivos de decrecimiento turístico inspirados en el altermundismo se insertan en un marco discursivo donde se advierten argumentos que oscilan desde posiciones semejantes a las más críticas del capitalismo social a otras que no contemplan posibilidad alguna de convivencia con las realidades que acogen las sociedades capitalistas (Fletcher, Murray, Blanco-Romero y Blázquez, 2019). Así, son reconocibles planteamientos que, entre la contención y el decrecimiento, abogan por la adopción de medidas orientadas a la no promoción de los destinos y por la revisión de las políticas fiscales con el fin de redistribuir beneficios y mitigar impactos —enfoque paliativo-, redimensionar la escala en la que se producen los movimientos turísticos, impulsando una transición hacia pautas cercanas al slow tourism -enfoque innovador- o el abandono de prácticas turísticas que impliquen el consumo de combustibles fósiles, al mismo tiempo que se reclama el desarrollo de infraestructuras turísticas ecológicas —enfoque reactivo-ambientalista-. Este último enfoque apostaría por una tercera vía que implica renunciar de alguna manera al turismo internacional como satisfactor de necesidades (Fernández-Miranda, 2013).

Estas ideas genéricas intentan armonizarse con medidas concretas como la regulación de los alojamientos turísticos, las moratorias de crecimiento, el uso de energías renovables y la restricción de los vehículos motorizados, la protección de los espacios naturales y los enclaves culturales, la aplicación de tasas al alojamiento y al desplazamiento de turistas o la limitación de la capacidad de carga de las infraestructuras de transporte y de los equipamientos de ocio. Aunque, como apunta Blanco-Romero (2019), resulta complicado actuar en estas direcciones sin provocar a continuación el encarecimiento - y la consiguiente elitización- de la oferta existente.

\section{SÍNTESIS COMPARATIVA}

A la luz de lo expuesto, se ofrece una síntesis que ordena los elementos constitutivos de cada una de las posiciones descritas. En las figuras 2, 3 y 4 se condensan las claves desde las que definen la realidad turística. 
Figura 2

\section{Discurso sobre la realidad turística}

\begin{tabular}{lll}
\hline & $\begin{array}{l}\text { Acción sobre la realidad } \\
\text { empírica }\end{array}$ & $\begin{array}{l}\text { Propuesta de realidad } \\
\text { alternativa }\end{array}$ \\
\hline Posición neoliberal & Aceleración & No existe \\
Posición del capitalismo social & Regulación & Desarrollo sostenible \\
Posición altermundista & Decrecimiento & Utópica \\
\hline
\end{tabular}

Fuente: elaboración propia

Figura 3

Estructura ideológica

\begin{tabular}{llll}
\hline & Diagnóstico & Pronóstico & Terapia \\
\hline Posición neoliberal & Buena salud & Positivo & No se requiere \\
Posición del capitalismo social & Problemas & Incierto & Medicación \\
Posición altermundista & Crisis & Colapso & Cambio de vida \\
\hline
\end{tabular}

Fuente: elaboración propia

Figura 4

Discurso sobre el derecho al turismo

\begin{tabular}{lll}
\hline & Derecho al turismo & Medio \\
\hline Posición neoliberal & Universal & Mercado \\
Posición del capitalismo social & Universal & Estado-Mercado \\
Posición altermundista & Cuestionable & Nuevo orden social \\
\hline
\end{tabular}

Fuente: elaboración propia

El liberalismo, conformado desde sus orígenes en coherencia con la filosofía empirista (Rasmussen, 2017), concibe la realidad como equivalente a la realidad accesible desde un punto de vista empírico. Cualquier alusión a lo que debería ser frente a lo que es daría pie a una entelequia mistificadora. Además, la realidad turística realmente existente es bien valorada y la única alternativa 
posible consistiría en intensificar las dinámicas que la producen, eliminando los impedimentos que puedan obstaculizar la incorporación del mayor número de individuos a la libre y natural acción del mercado —cristalización en el mundo moderno de la libertad de acción inherente al ser humano-.

Para la posición del capitalismo social, el análisis de las realidades que envuelven al sistema turístico obliga a una intervención en la que tendrían cabida diversas estrategias de colaboración entre administraciones públicas, empresas privadas y organizaciones ciudadanas. El fin coincide con el de la posición neoliberal: garantizar el negocio turístico internacional haciéndolo accesible a cuantas más personas mejor. Pero este objetivo solo podrá alcanzarse si se corrigen los impactos que genera el turismo, pues sin esos ajustes el futuro del sistema se torna incierto. En la revisión documental llevada a cabo, los textos encontrados con discursos susceptibles de ser clasificados como articulaciones ideológicas del capitalismo social han sido mayoritarios. Como se indicaba con anterioridad, excede a los objetivos planteados dilucidar qué tipo de mentalidad ideológica subyace a esta dinámica discursiva —esto es, esclarecer si quien produce el discurso se cree su contenido o si es el fruto de una impostura-.

Desde el altermundismo la realidad turística se evalúa de forma más crítica y la intervención sobre la misma exige una redefinición de los fines que estaríamos dispuestos a aceptar y de los medios que toleraríamos emplear. La razón que motiva esta brusca reorientación es la necesidad de concebir el turismo de manera diferente pues, de no hacerlo, la sociedad en su conjunto se vería abocada a un colapso ecológico. Debe aclararse que cuando en la figura 2 se tipifica la alternativa altermundista como utópica se hace en el sentido en el que Mannheim (1929) utilizó este concepto, para referirse a un modo de pensamiento cuya crítica del orden social existente va unida a la implantación de realidades que no existen y cuya existencia sería en gran medida incompatible con el mantenimiento de los pilares en los que se sostiene la realidad accesible en términos empíricos. Por supuesto, el propio Mannheim reconocía que era difícil precisar cuándo nos hallamos ante una propuesta que implique este tipo de desproporción porque, en definitiva, la historia está plagada de planteamientos etiquetados como utópicos que, con el tiempo, se acabaron convirtiendo en realidades tangibles. 
Figura 5

Tensiones

\begin{tabular}{lll}
\hline & Promesa & Principio de realidad \\
\hline Posición neoliberal & $\begin{array}{l}\text { Todos ganarán si confiamos } \\
\text { en el laissez faire. }\end{array}$ & Unos ganan y otros pierden. \\
Posición del capitalismo & $\begin{array}{l}\text { Con algunos ajustes, todo } \\
\text { mejorará. }\end{array}$ & $\begin{array}{l}\text { Las crisis no se podrán evitar. } \\
\text { si acaso, se podrán gestionar. }\end{array}$ \\
Posición altermundista & $\begin{array}{l}\text { Todo se transformará, o lo } \\
\text { pagaremos caro. }\end{array}$ & $\begin{array}{l}\text { La distancia hasta el nuevo } \\
\text { orden es muy grande. }\end{array}$ \\
\hline
\end{tabular}

Fuente: elaboración propia

La figura 5 resalta algunas de las tensiones a las que están expuestas las ideologías descritas. Las diferencias apuntadas entre la promesa y el principio de realidad forman parte de los factores que confieren a cada posición un carácter ideológico.

Para la posición neoliberal, el sistema turístico es capaz de autorregularse. Solo hay que confiar en la lógica mercantil del laissez faire. Los efectos de arrastre que el gasto turístico produce en otros sectores económicos — transporte, alimentación, construcción, etc.- dinamizan el tejido empresarial y crean riqueza. No importa si al principio esa riqueza se concentra en grupos concretos porque terminará empapando todo el entramado social. Esta promesa, como las de los otros dos bloques, contiene una parte de verdad, pero omite la existencia de un espacio sombrío: la dinámica del mercado está penetrada por relaciones de poder que, en ausencia de mecanismos socializadores de los beneficios y mitigadores de los costes, derivan a menudo en dinámicas de subordinación estructural de las regiones receptoras de turistas a los intereses de las élites gestoras del negocio turístico (Goldstone, 2001; Nowicka, 2008).

Los discursos del capitalismo social se fusionan con los de la sostenibilidad. Todo puede mejorar con ajustes y cierta regulación. El éxito de esta estrategia se aprecia en la influencia que ejerce en el entramado de organizaciones políticas y empresariales. La razón de su expansión reside en su carácter propagandístico. Es difícil superar una adecuada combinación de grandes dosis de esperanza aderezada por unas gotas de compromiso y autocrítica, sobre todo si el cóctel resultante no implica un cuestionamiento profundo de las lógicas dominantes y, en especial, del principio según el cual se asume que el turismo 
es un bien social a cuyo acceso tiene derecho todo el mundo. La dimensión ideológica de este modo de proceder tiene que ver con el hecho de no explicitar que el equilibrio socioambiental que se obtiene de la desigual aplicación de medidas dirigidas a promover el turismo sostenible es muy precario y no evita la proliferación de crisis sistémicas de diferente intensidad cuyos impactos, en el mejor de los casos, podrán gestionarse.

Finalmente, la posición altermundista advierte de la inviabilidad de mantener el negocio turístico internacional y, por lo tanto, de la necesidad de emprender un nuevo camino con una meta que, de momento, no se distingue en el horizonte, pero por la que valdría la pena luchar: la sustitución del actual orden político-económico, impulsor y legitimador de las prácticas turísticas hegemónicas, por otro tipo de orden donde la justicia social y el respeto por la naturaleza reemplace el interés económico como leitmotiv vital. En el nuevo orden el turismo se gestionaría según principios de decrecimiento o sería sustituido por otro tipo de actividades económicas que no generasen impactos tan agresivos. Esta promesa lleva asociada al menos dos problemas de unas dimensiones no comparables a las que plantean las otras dos ideologías: a) el esfuerzo que requiere la aplicación de la terapia es percibido como inaceptable por, quizá, demasiados actores del sistema turístico, y b) las pautas de consumo turístico criticadas desde esta posición como inasumibles están interiorizadas como no problemáticas por, quizá también, demasiados millones de personas.

\section{REFLEXIÓN FINAL}

La cuestión del derecho al turismo es difícil de abordar, teniendo en cuenta la complejidad adquirida por el sistema turístico y la variedad de casuísticas que integra. Las relaciones existentes entre las prácticas turísticas y sus interpretaciones subjetivas dan forma a múltiples y cambiantes contextos en los que las acciones y los discursos se influyen y retroalimentan constantemente.

El trabajo realizado ha servido para tipificar y comparar las posiciones de tres bloques ideológicos — neoliberal, del capitalismo social y altermundistaque pugnan por imponer su definición de la realidad turística y su valoración de lo que debería ser. A través de la identificación de los discursos emitidos por organizaciones clave en el sistema turístico se ha podido ahondar en la comprensión de los argumentos que legitiman o cuestionan la consideración del turismo como un derecho social. La explicación desemboca en la exposi- 
ción de algunas de las tensiones internas que arrastran cada uno de los discursos. Con todo, el objetivo general se ha alcanzado: ayudar a desenmarañar la madeja ideológica que rodea al turismo. Creemos que esta es una operación necesaria y útil para poder dirigir la investigación de los fundamentos éticopolíticos sobre los que se erigen los discursos que justifican el derecho al turismo.

El objeto de estudio ofrece muchas aristas. Al hilo de todo lo apuntado, una línea de trabajo interesante es la que resulta de profundizar en los procesos de construcción sociopolítica de los discursos sobre el vínculo entre el turismo y el medio ambiente. Entre los turistas que acechan prostitutas en Phuket, los que visitan museos en Madrid, aquellos que viajan en cruceros por el Caribe, los europeos que viven experiencias de turismo comunitario en Latinoamérica o los que son aficionados a los safaris por Kenia existen muchas diferencias, pero hay un denominador común relevante: la contaminación ligada a los desplazamientos.

Desde el punto de vista de las comunidades receptoras también debe tenerse en cuenta que, en ausencia de otras alternativas de desarrollo reconocibles o de un tejido asociativo capaz de articular acciones contestatarias, una relativa socialización de los beneficios económicos generados por el turismo puede coincidir con transformaciones en las estructuras socioculturales y en el espacio natural que sean legitimadas por amplios sectores de la propia comunidad, si estos perciben una mejora en las condiciones materiales en las que discurren sus vidas cotidianas (Mantecón, 2010, 2012). Esta idea ya había sido mencionada en un libro tan crítico como el de Goldstone (2001), donde la autora mostraba su escepticismo ante la posibilidad de que las poblaciones balinesas tradicionales reprodujeran la construcción de cabañas si tuviesen la oportunidad de vivir en casas de hormigón climatizadas.

Una variación un tanto distorsionada de esta discusión es la que desarrolla Butcher (2003), según la cual el ecoturismo y el turismo comunitario serían la proyección de una ideología moralizante que impide la expansión de los benéficos efectos que se derivan de la modernización. Higgins-Desbiolles (2018b) ha criticado la justificación que impregna este argumento. Probablemente Butcher tenga una parte de razón en la crítica que formula al ecoturismo y a otras formas de turismo etiquetadas por él como moralizantes, pero incurre en un problema que revela, como afirma Higgins-Desbiolles, su verdadera agenda ideológica: los inconvenientes que encuentra en las alternativas turísticas desarrolladas en los últimos años no justifican las pautas turísticas hegemónicas, más bien le sirven de excusa para legitimar la posición neoliberal. 
En fin, estudiar los aspectos ideológicos que rodean al hecho turístico es importante para analizar la dinámica de esta realidad y, en concreto, para comprender sus dimensiones éticas, pues, como explicaba Habermas (1973), si la perturbación del equilibrio ecológico indica el grado de explotación de los recursos naturales, lo que estamos dispuestos a tolerar los seres humanos no tiene límites precisos. En cambio, estos son redefinidos en contextos discursivos dinámicos donde los actores sociales implicados cuentan con desiguales recursos para tratar de imponer su definición de la situación.

\section{BIBLIOGRAFÍA}

Aguiló, Eugeni y Vich-i-Martorell, Gabriel (1996). La investigación en el ámbito de la política turística. Estudios Turísticos, 129, 23-35.

Artini, Luh-Putu, Nilan, Pam y Threadgold, Steven (2011). Young Indonesian Cruise Workers, Symbolic Violence and International Class Relations. Asian Social Science, 7(6), 3-14.

Ateljevic, Irena y Doorne, Stephen (2002). Representing New Zealand: Tourism Imagery and Ideology. Annals of Tourism Research, 29(3), 648-667.

Berger, Peter (1963). Invitation to Sociology. New York: Anchor.

Bianchi, Raoul y Stephenson, Marcus (2014). Tourism and citizenship. Abingdon: Routledge.

Bianchi, Raoul, Stephenson, Marcus y Hannam, Kevin (2020). The contradictory politics of the right to travel. Mobilities, $15(2), 290-$ 306.

Blanco-Romero, Asunción (2019). Decrecimiento turístico. Recuperado de: www.albasud.org [Consultado el 2 de febrero de 2020].

Blázquez, Macià (2014). Un pulso por el decrecimiento urbanoturístico. Via Tourism Review, 6.

Bramwell, Bill (2006). Actors, power and discourses of growth. Annals of Tourism Research, 33(4), 957-978.

Bramwell, Bill y Meyer, Dorothea (2007). Power and tourism policy relations in transition. Annals of Tourism Research, 34(3), 766-788. 
Breakey, Noreen y Breakey, Hugh (2013). Is there a right to tourism? Tourism Analysis, 18, 739-748.

Burns, Peter y Novelli, Marina (2007). Tourism and Politics. Oxford: Elsevier.

Butcher, Jim (2003). The Moralisation of Tourism. London: Routledge.

Cals, Joan (1974). Turismo y política turística en España. Barcelona: Ariel.

Cañada, Ernest (2018). Too precarious to be inclusive? Hotel maid employment in Spain. Tourism Geographies, 20(4), 653-674.

Castañeda, Quetzil (2012). The neoliberal imperative of tourism: Rights and legitimization in the UNWTO Code of Ethics for Tourism. Practicing Anthropology, 34(3), 47-51.

Clemente, Juan-Antonio, Bote, Marcos y Sánchez-Vera, Pedro (2018). El turismo social accesible como nuevo modelo turístico. Cuadernos de Turismo, 41, 139-159.

Cohen-Hattab, Kobi (2004). Zionism, Tourism, and the Battle for Palestine: Tourism as a Political-Propaganda Tool. Israel Studies, 9(1), 61-85.

Conde, Fernando (2009). Análisis sociológico del sistema de discursos. Madrid: CIS.

De Kadt, Emmanuel (1979). Tourism. Passport to Development? New York: Oxford University.

Esping-Andersen, Gøsta (1990). The Three Worlds of Welfare Capitalism. Cambridge: Polity.

Estivill, Jordi (1979). Lloret de Mar: destruccions i resistències d'un poble en mans del turisme. Papers. Revista de Sociologia, 10, 175-201. Exceltur (2020). Mensaje del presidente. Recuperado de: www.exceltur.org [Consultado el 6 de febrero de 2020].

Farreny, Ramón, Oliver-Sola, Jordi, Lamers, Machiel, Amelung, Bas, Gabarell, Xavier, Rieradevall, Joan, Boada, Martí y Benayas, Javier (2011). Carbon dioxide emissions of Antarctic tourism. Antarctic Science, $23(6), 55^{6-566 .}$ 
Fennell, David (2019). The Future of Ethics in Tourism. En FayósSolà, Eduardo y Cooper, Chris (Eds.). The Future of Tourism (155177). New York: Springer.

Fernández-Miranda, Rodrigo (2013). Los límites olvidados. Reflexiones sobre turismo global, sostenibilidad y decrecimiento. Recuperado de: www.albasud.org [Consultado el 2 de noviembre de 2018].

Fletcher, Robert, Murray, Ivan, Blanco-Romero, Asunción y Blázquez, Macià (2019). Tourism and degrowth: an emerging agenda for research and praxis. Journal of Sustainable Tourism, $27(12), 1745^{-1763 . ~}$

Foster, John Bellamy (2011). Capitalism and Degrowth: An Impossibility Theorem. Monthly Review, 62(8), 26-33.

Gascón, Jordi (2016). Deconstruyendo el derecho al turismo. Revista CIDOB d'Afers Internacionals, 113, 51-69.

Gascón, Jordi (2019). Tourism as a right: a «frivolous claim» against degrowth? Journal of Sustainable Tourism, 27(12), 1825-1838.

Gascón, Jordi y Cañada, Ernest (2017). El mundo es finito, también para el turismo. Del multiplicador turístico al conflicto redistributivo. Oikonomics, 7, 28-33.

Gascón, Jordi y Ojeda, Diana (2014). Turistas y campesinado. Madrid y Tenerife: FTR y Pasos.

Gaviria, Mario (Dir.) (1974). España a go-go. Turismo charter y neocolonialismo del espacio. Madrid: Turner.

Gil, Javier (2019). Redistribución económica y precariedad. El caso de los anfitriones de Airbnb. Recerca. Revista de Pensament $i$ Análisi, $24(1), 9^{2-113}$.

Gil de Arriba, Carmen (1992). Casas para baños de ola y balnearios marítimos en el litoral montañés, 1868-1936. Santander: Universidad de Cantabria.

Goldstone, Patricia (2001). Making the World Safe for Tourism. New Haven: Yale University. 
Greenpeace (2018). ¡Vacaciones por fin! Pero sin destrozar el medio ambiente. Recuperado de: https://es.greenpeace.org/es/ [Consultado el 10 de febrero de 2020].

Greenwood, Davydd (1977). Culture by the Pound: An Anthropological Perspective on Tourism as Cultural Commoditization. En Smith, Valene (Ed.). Hosts and Guests (171186). Philadelphia: University of Pennsylvania.

Habermas, Jürgen (1973). Legitimationsprobleme im Spätkapitalismus. Frankfurt: Suhrkamp.

Hall, C. Michael (1994). Tourism and Politics. Chichester: John Wiley \& Sons.

Higgins-Desbiolles, Freya (2018a). Sustainable tourism: Sustaining tourism or something more? Tourism Management Perspectives, 25, 157-160.

Higgins-Desbiolles, Freya (2018b). La posibilidad de crear justicia a través del turismo. Via. Tourism Review, 13.

Huete, Raquel (2019). La digitalización de la economía y el fin del turismo. En Álvarez-Sousa, Antonio, Mantecón, Alejandro y Puertas-Cañaveral, Inmaculada (Eds.). Sociología del turismo (299321). Madrid: CIS.

Huete, Raquel y Mantecón, Alejandro (2018). El auge de la turismofobia ¿hipótesis de investigación o ruido ideológico? Pasos, 16(1), 9-19.

Igoe, Jim, Neves, Katja y Brockington, Dan (2010). A Spectacular EcoTour around the Historic Bloc: Theorising the Convergence of Biodiversity Conservation and Capitalist Expansion. Antipode, $42(3), 486-512$.

Jeffreys, Sheila (1999). Globalizing sexual exploitation: sex tourism and the traffic in women. Leisure Studies, 18(3), 179-196.

Jurdao, Francisco (1979). España en venta. Madrid: Ayuso.

López-González, José Luis (2018). Del código ético a la convención internacional. Un panorama crítico de la Organización Mundial 
del Turismo desde la perspectiva del cosmopolitismo. Oxímora. Revista Internacional de Ética y Política, 12, 205-223.

Mannheim, Karl (1929). Ideologie und Utopie. Bonn: Cohen.

Mantecón, Alejandro (2008). La experiencia del turismo. Un estudio sociológico sobre el proceso turístico-residencial. Barcelona: Icaria.

Mantecón, Alejandro (2010). Tourist modernisation and social legitimation in Spain. International Journal of Tourism Research, $12(5), 617-626$.

Mantecón, Alejandro (2011). El proceso del turismo residencial. Análisis sociopolítico de los discursos públicos desde una perspectiva cualitativa. Empiria. Revista de Metodología de Ciencias Sociales, 21, 17-38.

Mantecón, Alejandro (2012). El nexo entre urbanización y turismo. Análisis de la opinión pública. Papers. Revista de Sociologia, 97(1), 249-272.

Matthews, Harry y Richter, Linda (1991). Political science and tourism. Annals of Tourism Research, 18(1), 120-135.

McCabe, Scott y Diekmann, Anya (2015). The rights to tourism: reflections on social tourism and human rights. Tourism Recreation Research, $40(2)$, 194-204.

Milano, Claudio (2017). Turismofobia: cuando el turismo entra en la agenda de los movimientos sociales. Marea urbana, 1.

Nash, Dennison (1977). Tourism as a Form of Imperialism. En Smith, Valene (Ed.). Hosts and Guests (37-52). Philadelphia: University of Pennsylvania.

Nowicka, Pamela (2008). Vacaciones en el paraíso. Barcelona: Intermón Oxfam.

OIT (2019). El turismo sostenible: un catalizador del desarrollo socioeconómico inclusivo y la reducción de la pobreza en las zonas rurales. Recuperado de: www.ilo.org [Consultado el 23 de enero de 2020].

OMT (2001). Código Ético Mundial para el Turismo. Recuperado de: www.unwto.org [Consultado el 17 de marzo de 2019]. 
ONU (1948). Declaración Universal de los Derechos Humanos Recuperado de: www.un.org [Consultado el 17 de marzo de 2019]. ONU (1966). Pacto Internacional de Derechos Económicos, Sociales y Culturales. Recuperado de: www.ohchr.org [Consultado el 17 de marzo de 2019].

Pack, Sasha (2006). Tourism and Dictatorship. Gordonsville: Palgrave.

Panko, Thomas y George, Babu (2012). Child sex tourism. Criminal Justice Studies, 25(1), 67-81.

Pardo-Abad, Carlos y Nieto-Codina, Aurelio (2015). Turismo antártico. Anales de Geografía, 35(1), 139-168.

Picard, Michel (1996). Bali: Cultural Tourism and Touristic Culture. Singapore: Archipelago.

Rasmussen, Dennis (2017). The Infidel and the Professor. Princeton: Princeton University.

Robinson, Richard, Martins, Antje, Solnet, David y Baum, Tom (2019). Sustaining precarity: critically examining tourism and employment. Journal of Sustainable Tourism, 27(7), 1008-1025.

RTI (2015). Carta Mundial del Turismo Sostenible +20. Recuperado de: http://cartamundialdeturismosostenible2015.com/ [Consultado el 14 de noviembre de 2019].

Rutty, Michelle, Gössling, Stefan, Scott, Daniel y Hall, Michael (2015). The global effects and impacts of tourism. En Hall, Michael, Gossling, Stefan y Scott, Daniel (Eds.). The Routledge Handbook of Tourism and Sustainability (36-63). London: Routledge.

Schenkel, Erica (2018). Turismo social en América Latina. Lurralde, 41, 93-113.

Secretaría de Estado de Turismo de España (2019). Directrices generales de la Estrategia de Turismo Sostenible de España 2030. Recuperado de: https://turismo.gob.es [Consultado el 1o de enero de 2020].

Sepúlveda-Sánchez, Alejandro y Airey, David (2017). La influencia de la ideología en la política turística española y sus enfoques. Pasos, $15(4), 9^{25}-941$. 
Smith, Estellie (1997). Hegemony and Elite Capital. En Chambers, Erve (Ed.). Tourism and Culture (199-214). New York: SUNY.

Tejedo, Pablo, Pertierra, Luis, Benayas, Javier y Boada, Martí (2011). Equilibrios sobre el hielo: una breve (pero completa) revisión del conocimiento sobre el impacto humano en la Antártida. Ecosistemas, 20(1), 69-86.

Tourism Manifesto (2015). Manifiesto Europeo del Turismo para el Crecimiento y el Empleo. Recuperado de: https://www.tourismmanifesto.eu [Consultado el 3 de enero de 2020].

Transforming Tourism. Tourism in the 2030 Agenda (2017). Recuperado de: www.transforming-tourism.org [Consultado el 12 de enero de 2020].

Tribe, John (2007). Critical tourism. En Ateljevic, Irena, Pritchard, Annette y Morgan, Nigel (Eds.). The Critical Turn in Tourism Studies (29-39). Oxford: Elsevier.

Turner, Louis y Ash, John (1975). The Golden Hordes. London: Constable.

UNEP (2019). Paradise lost? Travel and tourism industry takes aim at plastic pollution but more action needed. Recuperado de: www.unenvironment.org [Consultado el 12 de enero de 2020].

Van-den-Berghe, Pierre y Flores-Ochoa, Jorge (2000). Tourism and nativistic ideology in Cuzco, Peru. Annals of Tourism Research, $27(1), 7-26$.

Velasco, María (2016). Dynamics of Spanish tourism policy: the political system as a driver and policy instruments as indicators of change (1952-2015). International Journal of Tourism Policy, 6(3-4), $256-272$.

Webster, Craig e Ivanov, Stanislav (2016). Political ideologies as shapers of future tourism development, Journal of Tourism Futures, 2(2), 109-124. 